\title{
Clinical value of serum eosinophilic cationic protein assessment in children with inflammatory bowel disease
}

\author{
Andrzej Wędrychowicz' ${ }^{1}$ Przemysław Tomasik², Stanisław Pieczarkowski ${ }^{1}$, \\ Kinga Kowalska-Duplaga ${ }^{1}$, Zofia Grzenda-Adamek ${ }^{1}$, Krzysztof Fyderek ${ }^{1}$
}

1Department of Pediatrics, Gastroenterology and Nutrition, Polish-American Children's Hospital, Jagiellonian University Medical College, Krakow, Poland

2Department of Clinical Biochemistry, Polish-American Children's Hospital, Jagiellonian University Medical College, Krakow, Poland

Submitted: 19 June 2012

Accepted: 4 September 2012

Arch Med Sci 2014; 10, 6: 1142-1146

DOI: 10.5114/aoms.2013.34415

Copyright @ 2014 Termedia \& Banach

\section{Abstract}

Introduction: Eosinophils contribute to the pathogenesis of inflammatory bowel disease (IBD) in the intestine. Eosinophilic cationic protein (ECP) is one of the most important eosinophilic specific mediators released during activation. The aim of the study was to evaluate the clinical value of serum ECP determination in children with active and inactive IBD and its correlation with disease activity. Material and methods: There were 125 children with IBD ( 63 with Crohn's disease - CD, 44 with ulcerative colitis - UC, 18 indeterminate colitis - IC) enrolled in the study. Among them 83 children were in the active phase of the disease, while the remaining 42 were in remission. The control group consisted of 56 healthy children. The ECP was assessed three times in children with active IBD, at baseline and after 2 and 6 weeks of treatment and once in children with inactive IBD and controls using fluoroenzymeimmunoassays.

Results: We found elevated ECP at baseline in the total active IBD group when compared to the inactive IBD and control groups, decreasing during treatment. Serum ECP was also elevated in the active UC and CD groups when compared to the inactive $U C$ and CD groups, and correlated with clinical UC and CD activity $(R=0.57$ and $R=0.52, p<0.05$, respectively) and duration of the clinical manifestation in UC $(R=0.62, p<0.05)$ but not with the disease location in the gastrointestinal tract, or endoscopic and histopathological activity.

Conclusions: Evaluation of serum ECP in children with IBD may be useful in disease activity assessment at onset and during the treatment.

Key words: eosinophilic cationic protein, inflammatory bowel disease, children.

\section{Introduction}

Ulcerative colitis (UC), Crohn's disease (CD) and indeterminate colitis (IC) are chronic diseases of the gastrointestinal tract belonging to the inflammatory bowel diseases (IBD). Inflammatory bowel diseases are characterized by chronic course of the disorder and periodic relapses. Inflammatory lesions in UC and IC are located in the mucosa of the colon, but in $\mathrm{CD}$ they may be located in any region of the digestive tract. Inflammation of the intestinal tissue is characterized by the accumulation of inflammatory cells in the affected tissue and release of multiple inflammatory mediators into the extracellular space $[1,2]$.
Corresponding author:

Andrzej Wędrychowicz MD, PhD

Department of Pediatrics,

Gastroenterology and Nutrition

Polish-American

Children's Hospital

Jagiellonian University

Medical College

265 Wielicka St

30-663 Krakow, Poland

Phone: +48 1265820 11,

ext. 1763

Fax: +48 126581088

E-mail: miwedryc@cyf-kr.edu.pl 
Results of the latest research studies confirmed the multifactorial etiology of IBD, including both genetic and environmental factors, although the direct cause of IBD is still unknown [3, 4].

The incidence of IBD considerably increased in Western Europe and USA in children and adolescents during the last three decades while the age of manifestation of the disease symptoms decreased $[5,6]$. The results of the epidemiological studies carried out in the last years confirm similar trends in Poland [7, 8].

During the course of IBD, inflammatory lesions occur in the intestine, including oedema of the mucosa and ulcerations with depletion of the goblet cells and disturbance of mucus production [9]. In histopathological assessment of biopsy specimens of the inflamed mucosa, intensive inflammatory infiltrations composed of lymphocytes, neutrophils and eosinophils were described. Eosinophils also have the ability to migrate through the cells of the intestinal mucosa epithelium and form crypt abscesses in IBD [10]. Increased infiltrations of the eosinophils were reported in the mucosa of the colon in active UC patients when compared to patients in remission $[11,12]$.

Increased eosinophil infiltrations were also described in the lamina propria of mucosa and submucosa of the colon in CD patients [13]. The activation of eosinophils in the inflammatory reaction is closely related to the release of various inflammatory mediators. Eosinophilic cationic protein $(E C P)$ is one of the most important eosinophilicspecific mediators involved in the inflammatory response in IBD [14]. During the progression of the inflammatory process, ECP released locally in the inflamed mucosa is permeated to the circulation and has a systemic impact.

The detailed role of eosinophilic granulocytes and the mediators secreted by them in the course of IBD is still not fully clarified.

Disease activity assessment is very important in the long-term care and treatment of children with IBD, because of the chronic nature of the disease and occurring relapses.

For full assessment of the severity of the disease in the lumen of the gastrointestinal tract, endoscopy should be performed. However, because of its invasive nature and the high cost, there is a need to seek laboratory markers of disease activity, which could be reliable in assessment of the intensity of inflammatory lesions in the intestine.

There are no studies estimating the changes of serum concentrations of ECP in children with active and inactive IBD up to now.

The aim of our study was to evaluate the clinical value of determination of serum ECP concentrations in children with active and inactive form of $\mathrm{IBD}$ and their correlation with disease activity.

\section{Material and methods}

One hundred twenty-five children with IBD (59 girls, 66 boys, average age: 12.5 years, range: 2.8-18.0) were enrolled in the study, including 63 children with CD, 44 with UC and 18 with IC. There were 83 children with active IBD ( 42 with CD, 28 with UC and 13 with IC) and 42 with inactive IBD (21 with CD, 16 with UC and 5 with IC). The control group consisted of 56 healthy children (30 boys, 26 girls, average age: 11.3 years, range: $3.5-$ 18.0) with no complaints from the gastrointestinal tract.

The study was carried out in accordance with the guidelines of the Declaration of Helsinki. The study protocol was approved by the Jagiellonian University Bioethical Committee and informed consent to participate in the study was obtained from all patients' legal guardians and patients more than 16 years of age enrolled in the study.

The concentration of ECP in serum was determined three times in the acute phase of IBD, at the baseline and after 2 and 6 weeks of treatment, and once in children with IBD in remission and the control group, using commercially available fluoroenzymeimmunoassays (Unicap ECP, Pharmacia, Sweden).

The diagnosis of IBD was based on the Porto criteria [15]. The clinical activity of UC and IC was measured according to the modified Truelove-Witts severity index and the clinical activity of CD was measured with the pediatric Crohn's disease activity index (PCDAI) [16]. The endoscopic and histopathological activity was evaluated with the Roth endoscopic scale and the histopathological standard scale $[17,18]$. Additionally, the serological parameters perinuclear anti-neutrophil cytoplasmic antibodies (pANCA) and anti-Saccharomyces cerevisiae antibodies (ASCA) were assessed.

\section{Statistical analysis}

The statistical analysis was performed with Statistica 7.0 software (StatSoft, Tulsa, USA) using ANOVA and Scheffe's test. Correlation coefficients between variables were assessed using Pearson's linear correlation test. Serum ECP concentrations were expressed as mean values and standard deviations. Value of $p<0.05$ was considered statistically significant.

\section{Results}

Assessing the serum ECP concentrations in the total active IBD group, we found them elevated at baseline compared to the inactive IBD group and controls, decreasing after 2 and 6 weeks of the treatment (Figures 1, 2).

Assessing serum ECP concentrations in subgroups of active IBD children, we found them sta- 
tistically significantly elevated in the active UC and CD groups as compared to controls and inactive UC and CD groups, whereas serum ECP concentration in the active IC group was comparable to the control group. Additionally, serum ECP concentrations strongly decreased after 2 weeks and continuously slightly decreased after 6 weeks of the treatment in both active UC and CD groups, but increased in the active IC group (Table I).

The serum ECP concentrations in the active UC and $C D$ groups correlated with the clinical disease activity $(R=0.57$ and $R=0.52, p<0.05$, respectively) (Figures 3 and 4). They also correlated with the values of erythrocyte sedimentation rate (ESR) ( $R=0.60$ and $R=0.44, p<0.05$, respectively) but did not correlate with $C$-reactive protein values and serological parameters (pANCA and ASCA) (Table II).

Moreover, serum ECP concentrations correlated with the duration of clinical manifestation of disease $(R=0.61, p<0.05)$ only in the UC group and not in CD and IC groups (Figure 5 ).

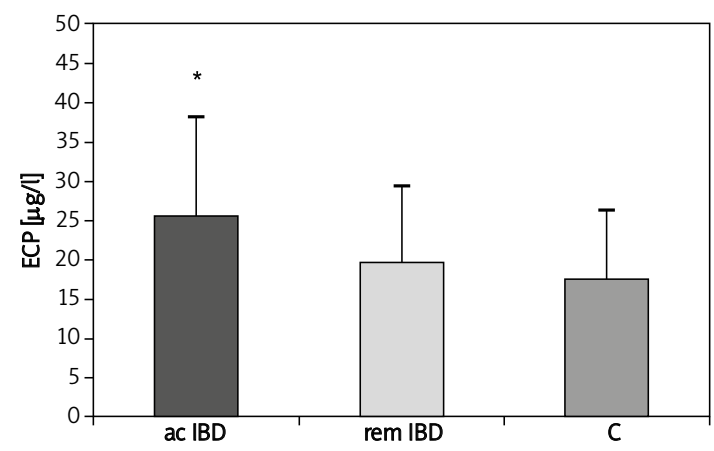

Figure 1. Serum ECP concentrations in active (ac IBD) and inactive IBD (rem IBD) and control (C) groups Values are expressed as mean and standard deviations; ${ }^{*} p<0.05$ for ac IBD vs. rem IBD and $C$.
There were also no statistically significant correlations between serum ECP concentrations and disease location in the gastrointestinal tract or with endoscopic and histological activity in UC, CD and IC groups.

\section{Discussion}

Eosinophil accumulation in the mucosa of the involved intestine is a frequent feature in different allergic and inflammatory disorders of the gastrointestinal tract [19].

The specific granules of the eosinophils contain a number of highly cationic proteins such as ECP, which has potent cytotoxic action and is released after activation and stimulation of the cells [20]. They play an important role in the development of inflammation in the gastrointestinal tract in the course of IBD [21]. The activation of eosinophils with increased release of ECP into the extracellular space and stool in adult patients both with UC and CD and their correlation with disease activity were reported [22, 23].

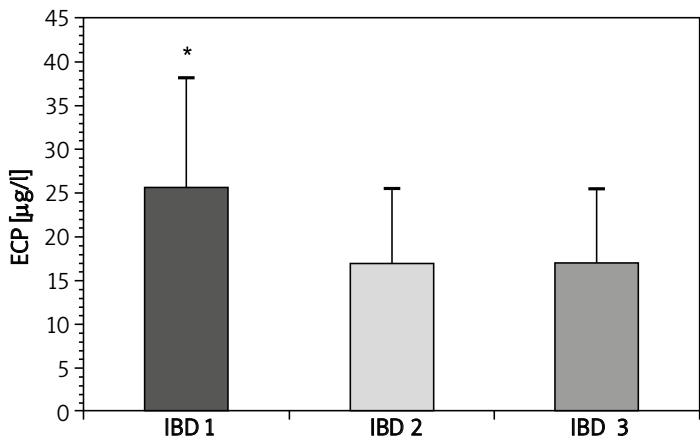

Figure 2. Serum ECP concentrations in total IBD group at baseline (IBD 1), and after 2 (IBD 2) and 6 (IBD 3) weeks of treatment Values are expressed as mean and standard deviations, ${ }^{*} p<0.05$ for active IBD 1 vs. IBD 2 and IBD 3.

Table I. Serum ECP concentrations in ulcerative colitis (UC), Crohn's disease (CD), indeterminate colitis (IC) and control $(C)$ groups during the treatment $(\mu \mathrm{g} / \mathrm{l})$

\begin{tabular}{|c|c|c|c|}
\hline Groups & Baseline & After 2 weeks & After 6 weeks \\
\hline \multicolumn{4}{|l|}{ UC: } \\
\hline Active $(n=28)$ & $25.31 \pm 15.42^{*}$ & $9.19 \pm 5.84^{*}$ & $6.80 \pm 4.86^{\star}$ \\
\hline Inactive $(n=16)$ & $15.82 \pm 11.36^{\star}$ & & \\
\hline \multicolumn{4}{|l|}{ CD: } \\
\hline Active $(n=42)$ & $26.91 \pm 13.95^{\star}$ & $16.31 \pm 8.38^{*}$ & $12.53 \pm 7.56^{\star}$ \\
\hline Inactive $(n=21)$ & $16.14 \pm 13.51^{*}$ & & \\
\hline \multicolumn{4}{|l|}{ IC: } \\
\hline Active $(n=13)$ & $18.80 \pm 11.25$ & $23.83 \pm 10.76$ & $24.09 \pm 12.54$ \\
\hline Inactive $(n=5)$ & $16.28 \pm 7.76$ & & \\
\hline \multicolumn{4}{|l|}{ C: } \\
\hline$(n=56)$ & $15.05 \pm 9.72^{\star}$ & & \\
\hline
\end{tabular}

Data expressed as mean values and standard deviations; ${ }^{*} p<0.05$ for baseline vs values after 2 and 6 weeks. 


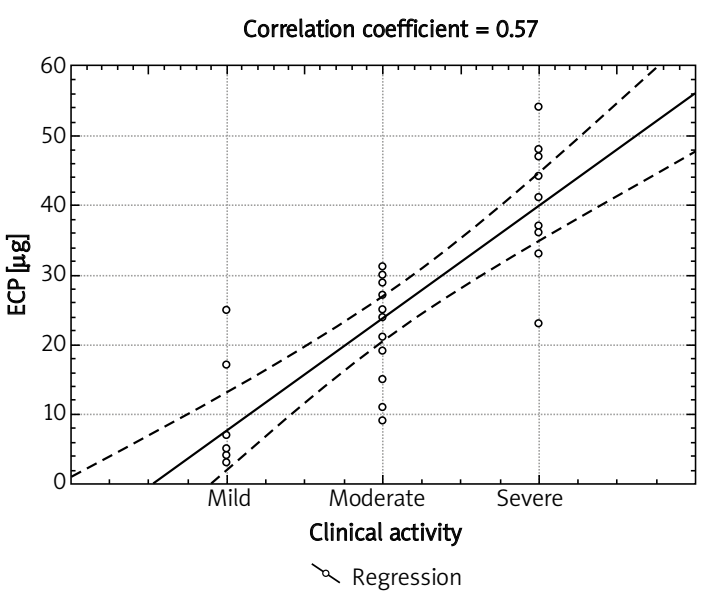

Figure 3. Correlation of serum ECP concentrations with clinical activity in UC group

Papers published in recent years confirmed activation of eosinophils in the pathogenesis of IBD both in animal model and in humans.

Shichijo et al. reported that using anti-ECP antibodies in the treatment of dextran sulfate sodiuminduced colitis in rats promoted intestinal wound healing and downregulated the immune response. Moreover, anti-ECP antibodies suppressed histopathological activity of the disease and increased the regenerative processes in the colonic epithelium [24].

There were no available studies assessing serum ECP in children with IBD. There are only a few clinical studies assessing ECP and its clinical value using different methodologies and different materials in children with IBD. Luck et al. assessing in vitro released ECP from peripheral blood eosinophils in children with IBD found increased concentrations of ECP in patients with active UC and CD when compared to patients in remission, but there were no differences between UC and CD with the same clinical activity of the disease. Additionally, the authors observed a significant correlation of ECP concentrations with the clinical activity of both UC and CD [12].

This observation is in concordance with our results. In our study we also found similar correlation ratios between serum ECP concentrations and the UC and CD clinical activities (0.57 vs. 0.52 ).

Troncone et al. assessed ECP concentrations in whole-gut lavage fluid from children with IBD. They also found significantly elevated ECP concentrations in UC when compared to CD and in both CD and UC children when compared to controls [25]. The method used by the authors required a whole-gut lavage with a nonabsorbable polyethylene glycolbased solution, which needed the patient's cooperation and was more invasive for pediatric patients.

There have been published several papers evaluating ECP concentrations and their correlation with disease activity in adult patients with IBD. Saitoh

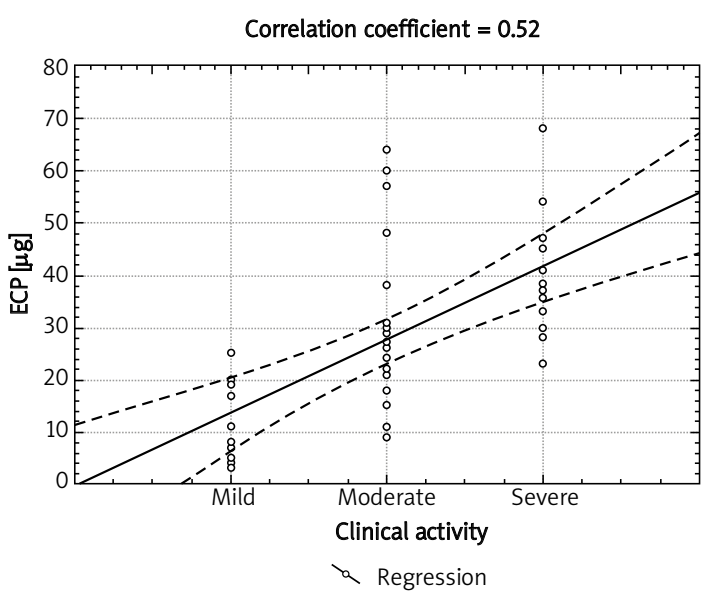

Figure 4. Correlation of serum ECP concentrations with clinical activity in CD group

Table II. Correlation of serum ECP concentrations with laboratory parameters in ulcerative colitis (UC) and Crohn's disease (CD) groups

\begin{tabular}{|lll|}
\hline Parameter & \multicolumn{1}{c|}{ UC } & \multicolumn{1}{c|}{ CD } \\
\hline ESR & $R=0.60, p<0.05$ & $R=0.44, p<0.05$ \\
\hline CRP & $R=0.42, p=\mathrm{NS}$ & $R=0.38, p=\mathrm{NS}$ \\
\hline $\mathrm{Hb}$ & $R=0.45, p=\mathrm{NS}$ & $R=0,31, p=\mathrm{NS}$ \\
\hline Albumin & $R=0.28, p=\mathrm{NS}$ & $R=0.41, p=\mathrm{NS}$ \\
\hline pANCA & $R=0.51, p=\mathrm{NS}$ & $R=0.43, p=\mathrm{NS}$ \\
\hline ASCA & $R=0.39, p=\mathrm{NS}$ & $R=0.47, p=\mathrm{NS}$ \\
\hline
\end{tabular}

$E S R$ - erythrocyte sedimentation rate, $C R P-C$ reactive protein, $\mathrm{Hb}$ - haemoglobin, pANCA - perinuclear anti-neutrophil cytoplasmic antibodies, ASCA - anti-Saccharomyces cerevisiae antibodies.

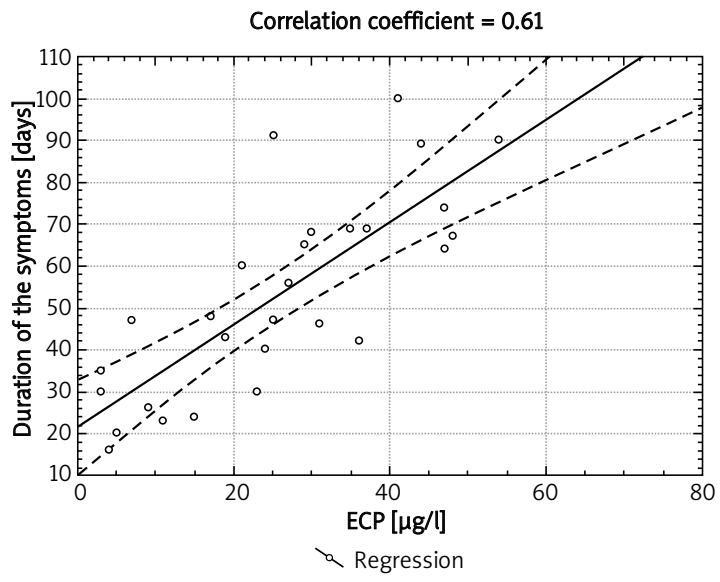

Figure 5. Correlation of serum ECP concentrations with duration of clinical manifestation in UC group

et al. assessed ECP concentrations in stool samples of CD and UC patients and controls. They observed significantly increased ECP concentrations in active CD and UC patients compared to inactive ones and controls. The authors also observed significantly increased concentrations of ECP within the inactive $U C$ and CD patients and also in UC and CD patients 
relapsing during the following 3 months compared to non-relapsing patients [23].

The results of the study of Raab et al. described the mucosal release of ECP in UC adult patients assessed by intraluminal segmental perfusion of the sigmoid colon and rectum, showing a severalfold increase of ECP when compared to controls. Moreover, ECP was markedly increased in the perfusate and related to the intensity of the inflammatory infiltrations in the mucosa [26].

Pronk-Admiraal et al. described elevated serum ECP concentrations in active UC adult patients compared to inactive ones and controls. Additionally, a decrease in serum ECP concentrations was accompanied by a decrease of blood eosinophil count and $\mathrm{C}$-reactive protein during pharmacological treatment in active UC [27].

The results of our study in IBD children are in concordance with earlier data in adults. They showed that ECP may play a similar role in the pathogenesis of UC and CD both in children and adults.

Our results showed increased serum ECP concentrations in active UC and CD children and adolescents as compared to inactive UC and CD patients and controls, decreasing during the treatment. Serum ECP concentrations correlated with the clinical disease activity and with ESR in both UC and $C D$ groups and with the duration of the clinical manifestation in the UC group.

In conclusion, serum ECP concentrations are different among active and inactive IBD patients and healthy children. They correlated with clinical activity of UC and CD and may be a useful inflammatory indicator of disease activity assessment in children with UC and CD, helping to evaluate and monitor the severity of the disease at onset and during the treatment.

\section{References}

1. Sartor RB. Cytokines in intestinal inflammation: pathophysiological and clinical considerations. Gastroenterology 1994; 106: 533-9.

2. Andoh A, Yagi Y, Shioya M, Nishida A, Tsujikawa T, Fujiyama Y. Mucosal cytokine network in inflammatory bowel disease. World J Gastroenterol 2008; 14: 5154-61.

3. Kaser A, Zeissig S, Blumberg RS. Genes and environment: how will our concepts on the pathophysiology of IBD develop in the future? Dig Dis Sci 2010; 28: 395-405.

4. Dudarewicz M, Baranska M, Skretkowicz J. Importance of selected polymorphisms of ABCB1 (MDR1) gene in inflammatory bowel diseases. Prz Gastroenterol 2010; 5: 310-4.

5. Lindberg E, Lindquist $B$, Holmquist L, Hildebrand $\mathrm{H}$. Inflammatory bowel disease in children and adolescents in Sweden, 1984-1995. J Pediatr Gastroenterol Nutr 2000; 30: 259-64.

6. Lee JC, Bridger S, McGregor C, Macpherson AJ, Jones JE. Why children with inflammatory bowel disease are diagnosed at a younger age than their affected parent? Gut 1999; 44: 808-11.

7. Jedynak-Wasowicz U, Wedrychowicz A, Przybyszewska K, et al. Epidemiology of ulcerative colitis in children in southern Poland, retrospective study 1992-2002. J Pediatr Gastroenterol Nutr 2004; 39 (Suppl. 1): S302.

8. Karolewska-Bochenek K, Lazowska-Przeorek I, Albrecht P, et al. Epidemiology of inflammatory bowel disease among children in Poland. Digestion 2009; 79: 121-9.

9. Fyderek K, Strus M, Kowalska-Duplaga K, et al. Mucosal bacterial microflora and mucus layer thickness in adolescents with inflammatory bowel disease. World J Gastroenterol 2009; 15: 5287-94.

10. Woodruff SA, Masterson JC, Fillon S, Robinson ZD, Furuta GT. Role of eosinophils in inflammatory bowel and gastrointestinal diseases. J Pediatr Gastroenterol Nutr 2011; 52: 650-61.

11. Levy AM, Kita K. The eosinophil in gut inflammation: Effector or director? Gastroenterology 1996; 110: 952-4.

12. Luck W, Becker M, Niggemann B, Wahn U. In vitro release of eosinophil cationic protein from peripheral eosinophils reflects disease activity in childhood Crohn disease and ulcerative colitis. Eur J Pediatr 1997; 156: 921-4.

13. Winterkamp S, Raithel M, Hahn EG. Secretion and tissue content of eosinophil cationic protein in Crohn's disease. J Clin Gastroenterol 2000; 30: 170-5.

14. Choy MY, Walker-Smith JA, Williams CB, MacDonald TT. Activated eosinophils in chronic inflammatory bowel disease. Lancet 1990; 336: 126-7.

15. IBD Working Group of the European Society for Paediatric Gastroenterology, Hepatology and Nutrition. Inflammatory bowel disease in children and adolescents: recommendations for diagnosis: the Porto criteria. J Pediatr Gastroenterol Nutr 2005; 41: 1-7.

16. Ryzko J, Socha J, Woynarowski M. Validation of disease activity score indexes for inflammatory bowel disease. Surg Childh Intern 1996; 1: 17-21.

17. Roth JLA. Ulcerative colitis. In: Gastroenterology. Bockus HL (ed.). WB Saunders, Philadelphia 1976; 645-749.

18. Cuvelier C, Barbatis C, Mielants H, De Vos M, Roels H, Veys $\mathrm{E}$. Histopathology of intestinal inflammation related to reactive arthritis. Gut 1987; 28: 394-401.

19. Rothenberg ME. Eosinophilia. N Engl J Med 1998; 338: 1592-600.

20. Gleich GJ, Adolphson CR. The eosinophilic leukocyte: structure and function. Adv Immunol 1986; 39: 177-253.

21. Walsh RE, Gaginella TS. The eosinophil in inflammatory bowel disease. Scand J Gastroenterol 1991; 26: 1217-24.

22. Makiyama K, Kanzaki S, Yamasaki K, Zea-Iriarte W, Tsuji Y. Activation of eosinophils in the pathophysiology of ulcerative colitis. J Gastroenterol 1995; 30 Suppl 8: 64-9.

23. Saitoh O, Kojima K, Sugi K, et al. Fecal eosinophil granulederived proteins reflect disease activity in inflammatory bowel disease. Am J Gastroenterol 1999; 94: 3513-20.

24. Shichijo K, Makiyama K, Wen CY, et al. Antibody to eosinophil cationic protein suppresses dextran sulfate sodium-induced colitis in rats. World J Gastroenterol 2005; 11: 4505-10.

25. Troncone R, Caputo N, Esposito V, et al. Increased concentrations of eosinophilic cationic protein in whole-gut lavage fluid from children with inflammatory bowel disease. J Pediatr Gastroenterol Nutr 1999; 28: 164-8.

26. Raab Y, Fredens K, Gerdin B, Hallgren R. Eosinophil activation in ulcerative colitis: studies on mucosal release and localization of eosinophil granule constituents. Dig Dis Sci 1998; 43: 1061-70.

27. Pronk-Admiraal CJ, Linskens RK, Van Bodegraven AA, Tuynman HA, Bartels PC. Serum eosinophil cationic protein in active and quiescent ulcerative colitis. Clin Chem Lab Med 2000; 38: 619-22. 